the particles together. They then machined the porous preform to generate the desired final shape.

Next, the authors heated the preform in a chemically reducing atmosphere (a mixture of $4 \%$ hydrogen in argon) at $1,100{ }^{\circ} \mathrm{C}$ and immersed it in a vat of liquid zirconium and copper $\left(\mathrm{Zr}_{2} \mathrm{Cu}\right)$ at the same temperature, before removing it and finally heating it at $1,350^{\circ} \mathrm{C}$. This process causes the zirconium to displace the tungsten from the tungsten carbide, producing zirconium carbide ( $\mathrm{ZrC})$, tungsten and copper. The liquid copper is forced out of the $\mathrm{ZrC}$ matrix as the material solidifies, so that the final object is formed of approximately 58\% ZrC ceramic and 36\% tungsten metal, with small amounts of residual tungsten carbide and copper. The beauty of the method is that the porous preform is converted into a non-porous $\mathrm{ZrC}$ /tungsten composite of the same dimensions (the overall volume change is approximately $1-2 \%$ ).

The clever manufacturing process is complemented by the robust properties of the final product. Caccia et al. find that, at $800^{\circ} \mathrm{C}$, the $\mathrm{ZrC/tungsten} \mathrm{cermet} \mathrm{conducts} \mathrm{heat} \mathrm{2.5-3} \mathrm{times}$ better than iron- or nickel-based alloys currently used in high-temperature heat exchangers - which should improve the effectiveness of such devices. Furthermore, the mechanical strength of the $\mathrm{ZrC} /$ tungsten cermet is higher than that of nickel-based alloys typically used in high-temperature applications, and is unaffected by temperatures up to at least $800^{\circ} \mathrm{C}$, even when the cermet had previously undergone 10 heating-cooling cycles between room temperature and $800^{\circ} \mathrm{C}$. By contrast, iron alloys (stainless steels) and nickel alloys lose $80 \%$ or more of their strength at temperatures between $500^{\circ} \mathrm{C}$ and $800^{\circ} \mathrm{C}$ (ref. 2 ).

Heat exchangers transfer the thermal energy generated by a power plant to the working fluid in a thermal engine (such as a steam turbine) that converts heat into mechanical energy. The mechanical energy in turn is used to generate electricity. The overall process of converting heat to electricity is known as a power cycle. The US Department of Energy, along with industrial partners, is currently building a 10-megawatt test facility for a power cycle that uses supercritical $\mathrm{CO}_{2}$ as the working fluid (see go.nature.com/2pi50mt). This power cycle promises lower costs and greater efficiency for future power plants, compared with currently used power cycles, but requires highly efficient heat exchangers. Caccia and colleagues' paper focuses on heat exchangers that could be used in this power cycle in concentrated solar power plants (which use sunlight concentrated by mirrors to generate electricity; Fig. 1), but the heat exchangers could also be used in advanced nuclear and fossil-fuel-fired power plants.

One technical challenge that must still be addressed concerns the oxidation resistance of the new cermet: the material is prone to oxidation in air at high temperatures such as might be experienced in a power-plant heat exchanger. Supercritical $\mathrm{CO}_{2}$ is only a weak oxidizing agent, but could still break down the cermet. Caccia et al. report that cermet oxidation can be prevented for up to 1,000 hours at $750{ }^{\circ} \mathrm{C}$ and at high pressure (20 megapascals) when the material is coated with a thin layer of copper, and if a small amount of carbon monoxide (50 parts per million) is mixed with the supercritical $\mathrm{CO}_{2}$. Nevertheless, long-term durability must still be proved.

Lastly, the authors' preliminary estimates indicate that the combined costs of raw materials and processing required to make a heat exchanger from the $\mathrm{ZrC} /$ tungsten cermet would be lower than for an analogous heat exchanger made from a conventional nickel alloy. Moreover, the cermet device would provide twice the power density - that is, it could be half the size of its nickel-alloy counterpart. The use of such heat exchangers might help to reduce the costs of renewable concentrated solar power, making it economically competitive with fossil-fuel-derived electricity.

Craig Turchi is in the Thermal Sciences Group at the National Renewable Energy Laboratory, Golden, Colorado 80401, USA. e-mail: craig.turchi@nrel.gov

1. Caccia, M. et al. Nature 562, 406-409 (2018).

2. ASME Boiler \& Pressure Vessel Code, Section II, Part D (2013)

\title{
CRISPR tool puts RNA on the record
}

The bacterial-defence system CRISPR-Cas can store DNA snippets that correspond to encountered viral RNA sequences. One such system has now been harnessed to record gene expression over time in bacteria. SEE ARTICLE P.380

\section{CHASE L. BEISEL}

$\mathrm{D}$ etermining the gene-expression profile of a cell is crucial to unlocking how its DNA blueprint gives rise to its physical characteristics and behaviours. The standard approach used currently involves RNA sequencing or single-cell imaging techniques that generate detailed snapshots of geneexpression profiles. However, these techniques capture such profiles only at the moment of analysis, and kill the cells. This makes it hard to capture fleeting gene-expression profiles or those that provide a complete picture of cells going through major behavioural or environmental changes. On page 380, Schmidt et al. ${ }^{1}$ report progress in overcoming this challenge by enlisting a bacterial-defence system that can create a DNA record of specific RNA sequences in a cell.

The CRISPR-Cas bacterial-defence systems are probably best known for their application in genetic engineering to cleave specific DNA sequences ${ }^{2}$. But another feature of these systems is the incorporation of snippets of DNA from unwanted intruders into a bacterium's own genome. These stored sequences provide a permanent 'memory' of infection, which can enable a defensive response if the same sequences are encountered again. The nucleotides are added to the cell's DNA in a configuration called a CRISPR array. The sequence of an array alternates between identical repeat sequences and the incorporated snippets, which are called spacers. As spacers are acquired, the array lengthens, and the positioning of spacers in the array reflects the order in which they were inserted ${ }^{3}$.

Almost all CRISPR-Cas systems acquire foreign genetic material by directly capturing DNA from an invader. Some previous work exploited this feature of CRISPR-Cas systems to record information in the form of acquired and stored nucleotide sequences. For instance, one approach ${ }^{4,5}$ used CRISPR-Cas-mediated acquisition of externally provided synthetic DNA to capture sequences in a specific order. The particular order of the nucleotides in the spacers was subsequently 'decoded' to link each CRISPR array to pixels in sequential images ${ }^{5}$. Another study ${ }^{6}$ used chemical cues from the environment to drive expression of a gene controlling the abundance of a form of circular DNA called a plasmid. As plasmid abundance rose in the cell, the plasmid became the preferred source of DNA snippets for new spacers; this linked the presence of the chemical cue to a stored spacer that matched the plasmid DNA. That study, in particular, set the stage for the use of CRISPR-Cas to record the expression of one or a few genes. Yet it was unclear how this approach could be extended to provide a comprehensive record of the geneexpression profile of a cell.

Schmidt and colleagues devised a creative solution by focusing on CRISPR-Cas systems that capture invading RNA rather than $\mathrm{DNA}^{7}$ (Fig. 1a). These systems need only two proteins to achieve this feat, with one protein making a DNA version of the RNA sequence 

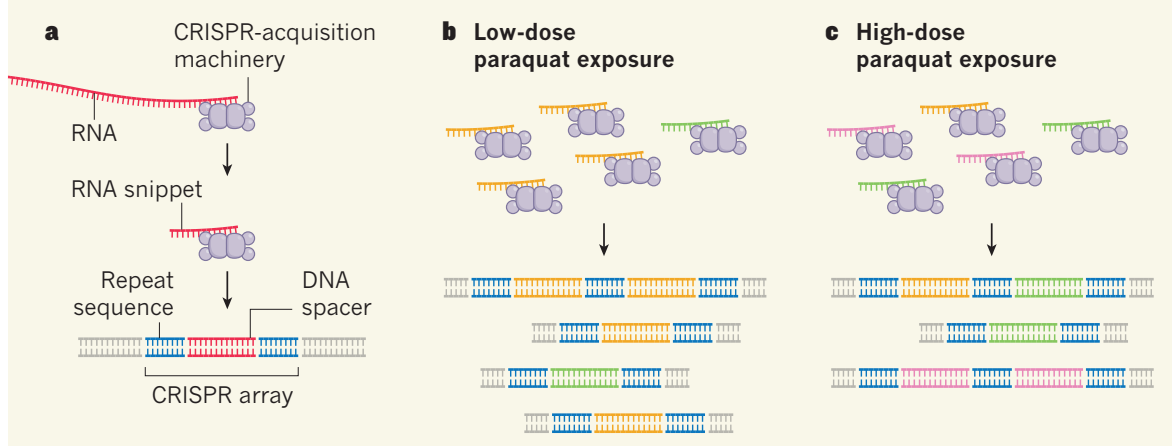

Figure 1 | A system to track RNA expression in cells. Schmidt et al. ${ }^{1}$ report the development of a technique to monitor gene expression by storing, and subsequently sequencing, DNA sequences that correspond to the RNA sequences expressed in bacterial cells. a, The authors engineered Escherichia coli bacteria to express CRISPR-acquisition machinery proteins from the bacterium Fusicatenibacter saccharivorans. These proteins can capture RNA transcripts and cleave a short snippet of RNA that is used to make a DNA version of the sequence - a spacer. This spacer is incorporated between repeat sequences of DNA to form a CRISPR array. b,c, The authors tested whether such captured DNA profiles could be used to document the abundance of RNA transcripts generated in response to different conditions, such as different dosages of the toxic molecule paraquat. They found that the technique could capture a 'fingerprint' of the gene-expression profile that was characteristic of the specific condition encountered by the cell.

that becomes the spacer. Being able to generate DNA from RNA raised the possibility that this DNA could be used to document the identity and abundance of RNA transcripts, and therefore capture a cell's gene-expression profile.

To use these CRISPR-Cas systems, the authors first had to overcome two technological hurdles. One hurdle was finding efficient RNA-capturing Cas proteins, because previously characterized proteins were inefficient at this task. The authors tested a large and genetically diverse set of Cas proteins, and identified clear winners from the human gut bacterium Fusicatenibacter saccharivorans. The other hurdle was being able to conduct DNA sequencing that focuses on the few CRISPR arrays that had obtained a new spacer, because most arrays were unaltered. The authors overcame this hurdle by developing a simple approach that selectively isolates the CRISPR arrays that have newly acquired spacers.

With these advances made, the authors went on to develop a method they called Record-seq for capturing gene-expression profiles. They genetically engineered the bacterium Escherichia coli to contain the RNA-acquisition proteins from F. saccharivorans. They then verified that these proteins could incorporate spacers into the genetic information of the E. coli cell, and that RNA rather than DNA sequences determined the corresponding spacer DNA.

In Record-seq, the RNA-acquisition proteins are expressed during the recording of the gene-expression profile. At the end of this period, a sample of the cell population is taken. Newly expanded CRISPR arrays are isolated and sequenced, and the spacers are matched to the corresponding genomic sequences.

The next steps were to prove that the method could faithfully create a record of gene expression and to determine what could be discerned about the cellular environment during the recording period. The authors found that Record-seq could record hundreds to thousands of different RNA transcripts present in the cell at any time. Although there was a strong bias towards capture of highly abundant transcripts, the transcript abundance of particular RNAs, as assessed by RNA sequencing, generally correlated with the frequency with which a corresponding spacer sequence was acquired in the sample. Furthermore, the collection of spacers could form a particular pattern depending on the growth conditions in which the cells were cultivated, allowing the authors to use such a spacer 'fingerprint' as a way to discern the conditions that the cells had experienced.

One key outcome was that the authors determined the characteristics that seem to govern the selection of RNA snippets (typically averaging around 40 base pairs in length) by the CRISPR-acquisition machinery during the process that generates spacers. Schmidt and colleagues found that the snippets were rich in adenine and thymine nucleotides, and often came from either one of the two ends of an RNA transcript. Unexpectedly, the authors found no obvious preference for specific sequences flanking the RNA regions used to make RNA snippets. Such flanking sequences, often termed protospacer-adjacent motifs (PAMs), are needed for the recognition process that enables CRISPR-Cas defences to specifically cleave the intended target sequence in the invader but not to cleave the same sequence present in the array $^{8}$. The system therefore might generate some spacers that will not enable an effective immune response to be launched because the corresponding target sequences are not flanked by a PAM. This possibility, and the ability of the RNA-acquisition proteins to acquire RNA snippets from the bacterium's own transcripts, raises questions about whether, and, if so, how, these systems effectively defend cells from unwanted intruders.
Arguably the most important demonstration of their method came when the authors compared Record-seq with direct sequencing of RNA. In one key experiment, the authors evaluated how well each technique could capture bacterial cells' transcriptional responses to a brief exposure to the toxic molecule paraquat. They found that only Record-seq could capture both transient and dosage-dependent features of the transcriptional response to paraquat exposure (Fig. 1b,c).

Schmidt and colleagues have laid the groundwork for using Record-seq to monitor complex gene-expression profiles over time, although there are some immediate technical limitations that must be overcome. One current limitation is that spacer acquisition still remains highly inefficient, requiring at least 10 million bacterial cells to faithfully record an expression profile. Another is that the authors tested their system only in bacterial cells, whereas much of the future potential of Record-seq might lie with animal and plant cells. Last, Record-seq was used to sequence arrays that have only one or two spacers, for reasons relating to how the newly expanded arrays were isolated and sequenced. If the technique is modified to analyse longer arrays, this could provide a way of discerning the timing and intensity of more than one cellular event during the same recording period. The successful application of DNA-based CRISPR technologies in various multicellular organisms, along with ongoing advances in the engineering of Cas proteins ${ }^{9-11}$, offer hope that Record-seq might overcome these challenges and eventually provide a robust and widely used technology.

As Record-seq is further developed, it might have many applications. Could it be used to track spatio-temporal changes in gene-expression profiles in multicellular systems and shed light on the development of animal and plant tissues and organs? Perhaps microbial communities in fluctuating micro-environments or the interactions between a pathogen and its host during infection could be monitored using this technique. Finally, will it be possible to use cells engineered to perform Record-seq to monitor gene expression in difficult-toaccess environments, such as the human gut, or to identify gene-expression profiles that are a signature of disease or abnormality? Schmidt and colleagues' technique might transform how gene-expression profiles are monitored in vivo in cells, and it highlights yet another aspect of CRISPR-Cas systems that can be harnessed to make powerful technologies.

Chase L. Beisel is at the Helmholtz Institute for RNA-based Infection Research and the University of Würzburg, 97080 Würzburg, Germany.

e-mail:chase.beisel@helmholtz-hiri.de

1. Schmidt, F., Cherepkova, M. Y. \& Platt, R. J. Nature 562, 380-385 (2018)

2. Barrangou, R. \& Doudna, J. A. Nature Biotechnol. 34, 933-941 (2016). 
3. Levy, A. et al. Nature 520, 505-510 (2015).

4. Shipman, S. L., Nivala, J., Macklis, J. D. \& Church, G. M. Science 353, aaf1175 (2016).

5. Shipman, S. L., Nivala, J., Macklis, J. D. \& Church, G. M. Nature 547, 345-349 (2017).
6. Sheth, R. U., Yim, S. S., Wu, F. L. \& Wang H. H. Science 358, 1457-1461 (2017)

7. Silas, S. et al. Science 351, aad4234 (2016)

8. Leenay, R. T. \& Beisel, C. L. J. Mol. Biol. 429, 177-191 (2017)
9. Kleinstiver, B. P. et al. Nature 523, 481-485 (2015). 10.Slaymaker, I. M. et al. Science 351, 84-88 (2016). 11.Hu, J. H. et al. Nature 556, 57-63 (2018).

This article was published online on 3 October 2018.

\title{
In Retrospect
}

\section{Method for studying dark matter turns 25}

\begin{abstract}
In 1993, two papers reported observations of an astronomical phenomenon called gravitational microlensing. The results showed that microlensing could be used to probe the elusive dark matter that is thought to pervade the Universe.
\end{abstract}

\section{GRZEGORZ PIETRZYŃSKI}

$\mathrm{O}$ ne of the biggest mysteries in astronomy is the nature of dark matter, which is thought to account for about $85 \%$ of the matter and $25 \%$ of the total energy in the Universe ${ }^{1}$. There are several strong pieces of evidence for dark matter ${ }^{2}$. In particular, spiral galaxies such as the Milky Way have flat rotation curves ${ }^{3}-$ graphs that show the orbital speed of stars as a function of their distance from the galactic centre. This property indicates that spiral galaxies are surrounded by large quantities of unseen matter. Dark matter has not yet been detected directly, so its identity is still unknown. But 25 years ago, Alcock et al. ${ }^{4}$ and Aubourg et al. ${ }^{5}$ reported observations in Nature that paved the way to a better understanding of its properties.

In 1986, the Polish astronomer Bohdan Paczyński suggested an observational test ${ }^{6}$ to determine whether dark matter present in the halo of our Galaxy is composed of astronomical bodies such as small stars, brown dwarfs, neutron stars or black holes. Such bodies are intrinsically faint and would therefore be difficult to see in the Galactic halo from Earth.

According to Einstein's general theory of relativity, these massive compact halo objects (MACHOs) could act as lenses, focusing light and amplifying the observed brightness of stars in nearby galaxies (Fig. 1). This phenomenon, called gravitational microlensing, is sensitive to even low-mass lenses. Paczyński therefore proposed that, by monitoring stars in nearby galaxies, astronomers could look for microlensing events and verify whether MACHOs can account for dark matter.

Conceptually, this test is simple. But in practice, it requires millions of stars to be monitored over a period of years. The reason is that the microlensing events have an extremely low probability of being detected, because the star, the MACHO and the observer all need to be aligned. At that time, it was a challenge to observe such a huge number of stars, accurately measure their brightness and analyse the resulting data.

In October 1993, Alcock et al. and Aubourg et al. independently announced the first candidates for microlensing events caused by dark objects in the Galactic halo. Alcock and colleagues used a dedicated 1.27-metre-diameter telescope. The telescope was equipped with two charge-coupled device (CCD) cameras that had a field of view of 0.5 square degrees, which was considered large at that time. The authors spent a year monitoring 1.8 million stars in a nearby galaxy known as the Large Magellanic Cloud, and discovered one microlensing candidate. By contrast, Aubourg and colleagues used photographic plates that had

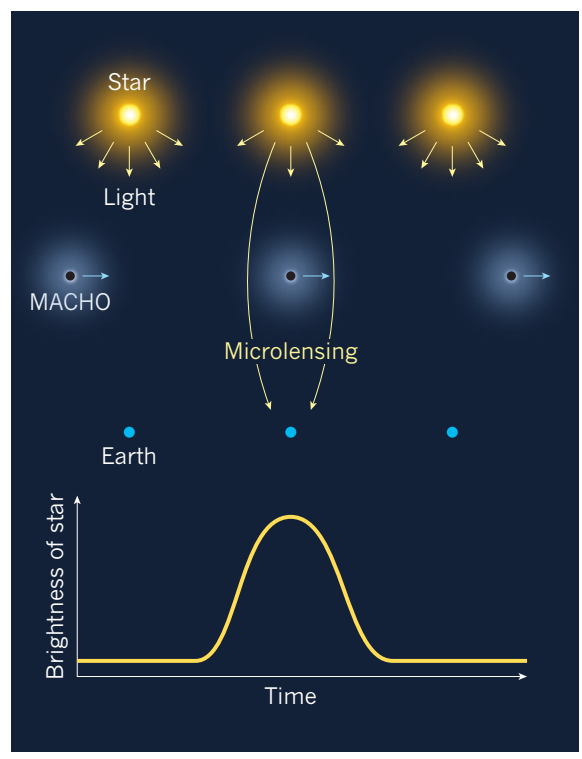

Figure 1 | Gravitational microlensing. In 1993, Alcock et al. ${ }^{4}$ and Aubourg et al. ${ }^{5}$ presented possible evidence for astronomical bodies called massive compact halo objects (MACHOs). Such bodies could account for dark matter - the 'missing' matter in the Universe. A MACHO can be detected when it passes in front of a star in a nearby galaxy. The MACHO bends light from the star towards Earth, which temporarily amplifies the star's observed brightness. This effect is known as gravitational microlensing. a field of view of about 25 square degrees. They monitored 3 million stars in the Large Magellanic Cloud for more than three years, and detected two microlensing candidates.

The candidates all had fairly symmetrical light curves - plots that show the observed brightness of a star as a function of time (Fig. 1). Both teams obtained light curves in two different colours (blue and red) and found that the shapes of these plots were extremely similar. Such symmetrical and achromatic light curves are in agreement with what is expected for microlensing events.

However, the light curves of the two candidates obtained using photographic plates had a low signal-to-noise ratio. And for all three candidates, there was incomplete coverage of the brightening event, especially close to the peak brightness. Furthermore, although the frequency of the observed candidates was consistent with theoretical predictions, their low number prevented any firm confirmation that the light curves were products of microlensing - rather than light curves of a previously unseen class of astronomical object of varying brightness.

Despite these limitations, the potential discovery of microlensing by dark objects in the Galactic halo was spectacular. It demonstrated the feasibility of using microlensing to detect extremely faint stellar-mass or sub-stellar-mass bodies in the Galactic halo and, as a result, the feasibility of verifying whether dark matter in spiral galaxies is composed of such bodies. The microlensing surveys showed that it was possible to monitor millions of stars over years and to analyse the resulting enormous data sets, which was a breakthrough in observational astronomy.

Motivated by the work of Alcock et al. and Aubourg et al., microlensing surveys improved enormously over the following 25 years, reaching capabilities to observe about 1 billion stars per night ${ }^{7}$. The huge data sets of high-quality light-curve data revolutionized many fields of astronomy - for example, the study of pulsating stars, extrasolar planets and star formation $^{8,9}$. Moreover, millions of objects of varying brightness and thousands of microlensing events were detected, mostly in the direction of the Galactic bulge (a spherical structure near the centre of our Galaxy).

However, on the basis of observations of 35 million stars over eight years, only four microlensing events in the direction of the Large Magellanic Cloud were detected ${ }^{10}$. If these signals were caused by MACHOs, the contribution of these objects to the mass of the Galactic halo must be low (a few per cent). But the more likely explanation of these detections does not involve MACHOs at all, and relies on the lensing of stars in the Large Magellanic 